\title{
Towards increased energy efficiency in industry - a manager’s perspective
}

\author{
Per-Erik Johansson ${ }^{1, *}$, Patrik Thollander ${ }^{2}$, Bahram Moshfegh ${ }^{2}$ \\ ${ }^{1}$ DynaMate Industrial Services AB, Stockholm, Sweden \\ ${ }^{2}$ Department of Management and Engineering, Linköping University, Linköping , Sweden \\ * Corresponding author. Tel: +46 0852293373, Fax: +46 0852293373, \\ E-mail: per-erik.johansson@dynamate-is.se
}

\begin{abstract}
Industry is one of the major users of fossils fuels resulting in emissions of GHG (Green House Gases), leading to global climate change. One means of promoting energy efficiency in industry is energy management. The aim of this paper is to outline a number of energy management related factors which affects energy management in industry positively. The paper is a result of collaboration between industry professionals and researchers within an ongoing research project and addresses the issue using a bottom-up energy management perspective. Results indicate that that the "soft" issues of energy management play a crucial role in the success (or not) of energy management in industry, e.g. the manager's role and attitude towards the employees cannot be understated. Instead it addresses that implementation is not only about technology but equally or even more important, concerns the diffusion and adoption of energy management practices and principals.
\end{abstract}

Keywords: Industrial energy management, Organizational change, Industrial energy efficiency

\section{Introduction}

Research indicates that global climate change resulting from the use of fossil fuels is one of the major challenges for future decision makers worldwide. Industry is one of the major users of fossils fuels resulting in emissions of GHG (Green House Gases), leading to global climate change. EU and other regions are now working proactively to reduce GHG emissions resulting from use of energy. One example is the 20-20-20-targets within the EU which in relation to energy means that each EU Member States should reduce the use of energy with $20 \%$ by reducing energy intensity with $3.3 \%$ annually from 2005 to 2020 . Industrial energy efficiency is one of the most efficient means of reducing GHG [1]. However, a number of barriers to energy efficiency exist in industry which inhibits adoption of energy efficient technologies and energy conservation [2-4]. One means of promoting energy efficiency in industry overcoming a number of barriers to energy efficiency is energy management [5-6]. Even though the potential is vast, research in the area is scarce [7]. One reason for this is its interdisciplinary character calling for interdisciplinary methods such as collaboration between researchers and industry professionals. The aim of this paper is to outline a number of energy management related factors which affects energy management in industry positively. The paper is a result of collaboration between industry professionals and researchers within an ongoing research project within Swedish industry and addresses the issue of promoting energy efficiency using a bottom-up energy management perspective. This paper is unique in the sense that is leaves the realm of focusing solely on energy efficient technologies when studying industrial energy efficiency.

\section{Methodology}

Previously, research has focused on energy management practices using questionnaires and in-depth interviews, e.g. [5] and [7]. In this study, the scope is to try to take the research on energy management in industry a leap further. Moving beyond questionnaire and in-depth interviews, an attempt is made to incorporate the manager's own ideas and concepts, not merely study an array of factors (using a questionnaire) or respondents' views and opinions (using interviews) on various themes or topics. As the applied method is narrowed down to 
fewer respondents, it naturally may be more difficult to generalize results from this type of research, see Fig 1. A previous literature review on energy management [7], shows that this research, so far is lacking in the academic literature, as well as clear results on how to apply successful energy management practices. We therefore conclude that this type of methodological approach is needed.

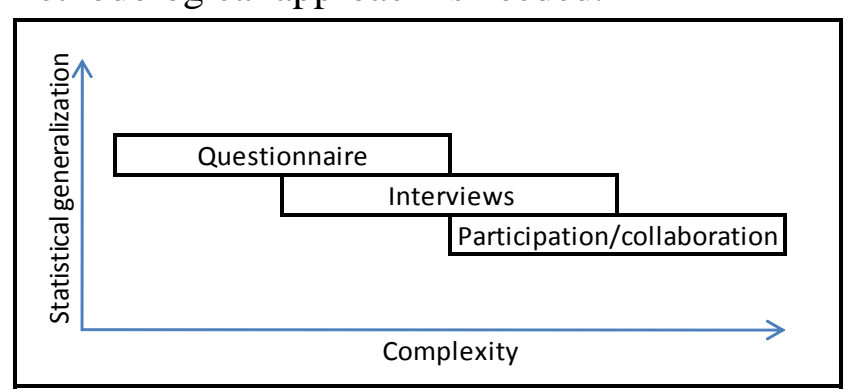

Fig. 1: Differences between participation/collaboration and interviews and questionnaires (Inspired by $[8]$.

According to [8], systems may be categorized depending on degree of complexity. [8] state nine levels and write that research concerning social interaction, i.e. interaction between two individuals is the most complex system to study. He moreover criticizes management research for not moving beyond the lower system levels. In response to [8]'s critique on management research, we aim to move beyond the more common methodological approaches using questionnaires and interviews applying participation/collaboration. Naturally, this limits the ability to generalize results, compared with questionnaires and interviews, as these methods cover several respondents. On the contrary, participation/collaboration methodology enables research to move into the more complex levels of (energy) management. This paper has applied a participation/collaboration methodology where the researchers have collected the manager's ideas and philosophies of successful energy management practices, based on more than 20 years of experience in the field. In doing so, it is evident that this relies on relatively a few cases, and naturally faces an increased risk of being biased, compared with questionnaires and interviews. When analyzing results from this paper, it is therefore important to keep in mind the statement by [5] "there is no one size fits all" when it comes to energy management.

\section{Results - driving forces for successful energy management practices}

In the following section, a number of important factor for successful organizational change related to energy management practices are presented. The stated factors come from the participation/collaboration approach, i.e. a bottom-up energy manager's perspective, a perspective which is derived from the truck manufacturer Scania's more than 20 years of successful energy management practices.

\subsection{Culture}

An organization has its own culture which is created by a number of factors, such as individuals own values [4]. Culture is an important factor when striving to change an organization, as culture governs the behavior of the individuals of the group, and it is their behavior that creates the organization's results. If any major changes are to take place within the organization, the behavior among the individuals has to change. In the long run, this will change the organizational culture, a change which is needed, if the individuals within the organization are to maintain their changed behavior. 


\subsection{Will}

The second factor of importance is that normally, people want to change things they are not satisfied with, while maintain that which one is satisfied with. The will to change is thus dependent on how dissatisfied a person is, and also how risk averse a person is. The importance for those who lead the change is to perceive that the challenge lies in that person's want the change to take place in their own way.

\subsection{Acceptance and recognition}

The third factor of importance is that people in general wants attention and positive recognition from personal achievements, from other persons. Many people can go a long way to receive recognition and acceptance. It can range anywhere from fame and compensation in the form of money for the effort one has made, to encouragement and a "thank you" for a good result that the person has accomplished. The former can never replace the encouragement from a manager, thanking the employee for good work. This holds in particular if the manager is also the informal leader, i.e. someone the employees look up to.

In general, people go to their work with a goal to be part of it as they wants to do a good job. If not, this creates discomfort, discouragement and frustration that often take the expression in unwillingness to cooperate, or an unwillingness to change situations at work. Paradoxically, people tend to do exactly the opposite of what it takes to get what one really wants. What this behavior gives in return is attention and an opportunity to be seen in the absence of recognition. Reasons why such an individual have lost sight can be, e.g. lack of attention, challenge or admission from those who are managers. It may also be due to an inadequate role and mission in the group the individual belong to. One may call this a deficiency in the organization caused by poor leadership.

The above three factors, culture, will and acceptance and recognition interact between each other and create the conditions one have in an organization, department and group. It is these challenges that the leader has to work with in order to achieve the expected results.

\subsection{Establishing change within an organization}

When a manager is leading a change in the organization, he or she can choose one of two main roads or paths. The difference between these two paths can be described by the following: To get from location A to B can be done in two principally different ways. The first option is to run in the sand at the water's edge. Although the road is long, it goes relatively quickly. The tracks in the sand are washed away rapidly and soon, no one else can, by the help of the first person's achievement, manage the very same way. Instead, each one has to take its own way to position B. The load to be moved from A to B depends on the individual's capacity and external conditions and circumstances. In summary, the first path is that of individuality, a path which does not help or support the persons that later wants to take the same path.

The second option is to build a road. It will take much more time, demands much more resources and effort, but when it is finished, there are clearly more people being able to travel from position A to position B. Moreover, people can get more loads with them on the road. The modes of transport can also be developed so that more cargo than was previously possible to carry can be included in each trip. The time to carry out the shipment may eventually be reduced. The load to be moved from A to B is through a road much less dependent on the individual's capacity and external conditions. In summary, the second path is that of the 
standardization and continuous improvement, a path which help and support the persons that later wants to take the same path.

Road number one may be stated as to govern by results. This is a road which with the right leadership often creates positive results relatively quickly, but the lasting result is often not maintained. The way to influence behavior is by getting members of the group to do what they request. As the manager does not require how results are achieved, solutions often rely upon individual solutions, i.e. the employees own way. Moreover, duplication of these individual solutions is generally not possible. This, in turn, leads to the fact that structural capital is not being built up within the organization, department or group. The culture is affected only to a limited extent and the impact it does create takes time. There is great risks that if the leader loses focus, or change job, the good results will not last.

Road number two is to use what we define as method governing. This is about influencing the behavior by using good methods and approaches. The modified behavior provides better results both in the economic sense, but also regarding the conditions to do a good job of maintaining or improving quality and work environment. The positive change one gains may be linked to the group and the positive spiral which then creates the opportunity to influence the culture of the group. As method governing focuses on how the work is done, conditions for working with continuous improvement are established.

Things which can be improved are the methods, routines and instructions. These are always the same; everyone in the group performs the operations in a similar way and can contribute to the improvement of the method. This will benefit both the individual and the group, even if the work and the physical and psychological conditions of the work are continuously changing. Method governing thus, and unlike the first road, builds structure capital, and with the right leadership, in the long run, an improvement in the culture of the group is achieved. This also reduces the risk of a manager changing job. However, and this must not be understated, bad leadership will always be able to bring down an organization, independent on which road that is taken.

\subsection{Successful organizational change}

Fig. 2. displays how an organization may successfully be transitioned by the second road, method governing. If one choose to work after the second road, there are some fundamental principles that should be followed, e.g. focus on the organization's value stream and continuous improvements. These are presented in the coming sections.

\subsection{Basis - the organization's value stream}

Start from the company's product's value stream and the company's services and do not view energy management as a means which creates value by itself. All work which is done in the organization (be it external or internal) must be valued based on the product's value stream. This provides the ability to sort and evaluate, not based on function, but on value. What is waste and what is value? Waste can also be divided into that which is necessary but not valueadding and that which is simply waste. What is value adding is often of no reason to attack first. It only involves the risk of new losses.

That which is necessary but not value adding should be minimized. That which is solely waste must be eliminated as quickly and smoothly as possible. It usually does not cost so much, it is often achieved relatively quickly, and the risk is often low. 


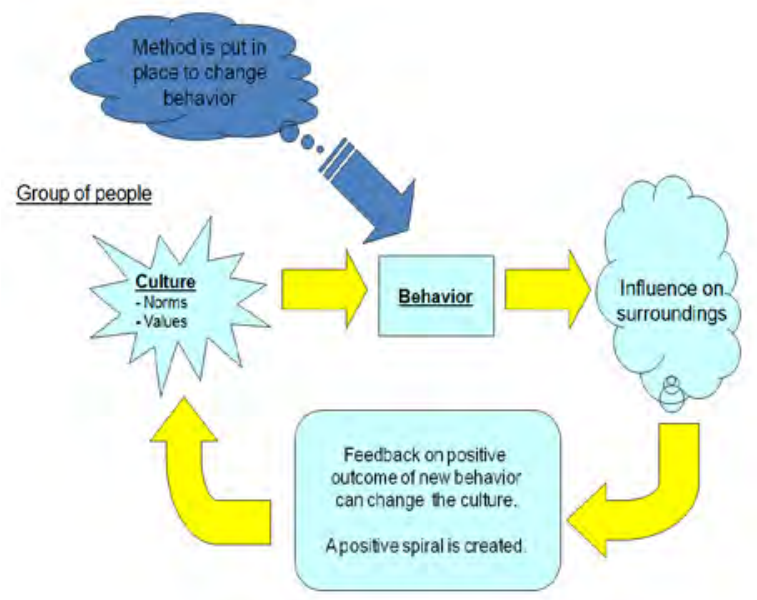

Fig. 2. Example of how an organization may be transitioned successfully based on method governing.

If the organization is a manufacturing company, it is important to understand how the production technologies, maintenance and energy management work is related. Increasing process efficiency is an effective way to reduce energy use for the product. To have a system perspective is necessary when improvements are carried out.

Moreover, aiming to achieve stable systems is of great importance. If for example, the shutdown of a machine or a process, leads to problems when the machine or process is being started, i.e. it may not work properly after shutting it down, employees may be unwilling to shutdown equipment. The root cause to this is not a system which is impossible to change, but rather an unstable system.

The eight types of waste which are relevant also in terms of industrial energy efficiency are: overproduction, unnecessary operations, transportation, discards, waiting, unnecessary movement, storage, and unused skills. Working to minimize and eliminate these both in the organization's value stream, but also in regard to the organizations energy use.

\subsection{Basis - continuous improvements}

The basis for long-term success is to work according to the principles of continuous improvement. This means that the leader must create an improvement culture within the group/company. The engine in the process of improvement is improvement groups and a systematic work with deviation.

To create an organization in which the principles of continuous improvement are used, and create a continuous improvement cycle, will not be made without effort. It demands leadership in order to be formed, kept active, and further developed. Fig. 3. visualize the above presented approach.

Work with improvement in small steps is of great importance, and one should aim to use all the tools for continuous improvement at disposal, such as deviation control. In order to be able to improve a system, one must be able to describe what the normal state or level is. If one does not know what the normal level is, improvements are not likely to take place. What is described and perceived as normal in the organization is what one can expect. When the outcome deviates from the expected normal state, the organization receives a signal that something has gone wrong. Detecing deviations may be achieved from, e.g. deviation management methods, the use of standards and routines etc. When a deviation from the 
normal state has been detected, the system can be improved in small steps, which in turn slowly increase the normal state level.

\section{Continuous improvement}

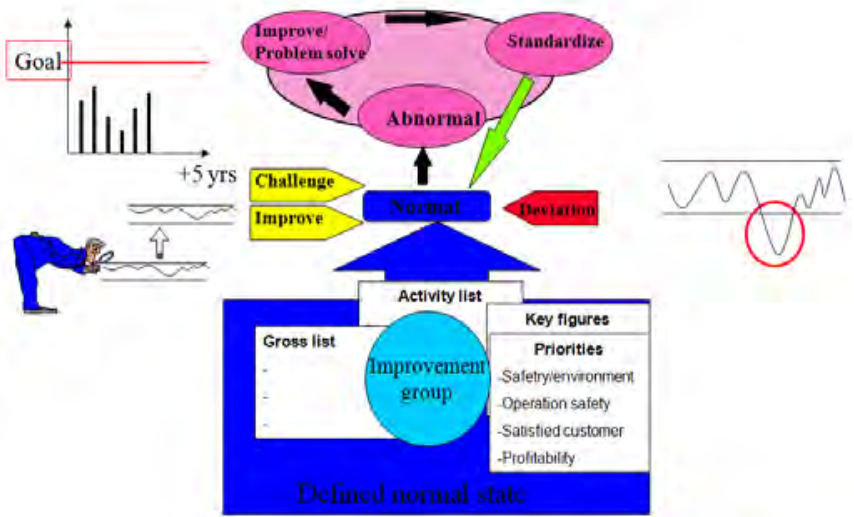

Fig. 3: Principles for continuous improvement in regard to energy efficiency.

Identify where the organization stand in terms of the performance it delivers. All phases have different needs for improvement. Improve from the defined normal state, do it with small steps but take steps often. Fig. 4. visualize the above presented approach.

\section{Normal state}

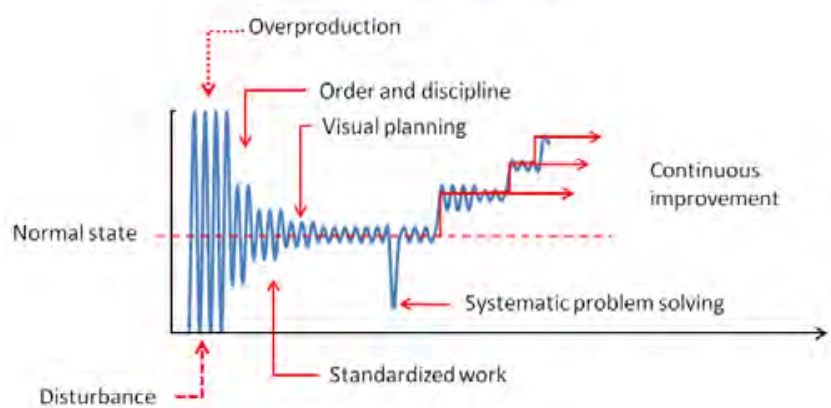

Fig. 4: Principles of defining and working with continuous improvement based on a normal state.

Ending improvement is as important as initiating them. Aim to try to find effective methods to evaluate when an improvement is "effective enough". Describe the new normal mode, implement it, and move on to the next improvement that gives the best effect on the overall efficiency.

Moreover, one should put focus on the flow (creating smooth flows, governed by demand, be sure to reduce the variance). Also, do not forget the work- and information flow. Otherwise, adequate conditions for improvements will not be established. Furthermore, one should focus on quality and time, and make sure to keep the correct delivery parameters, i.e. avoid variation and overproduction. Make sure to deliver on time and let demand govern as much as possible. By describing what is normal, delivery can be assessed, and the root cause for the deviation can be corrected.

Some general issues related to successful energy management practices are similar, no matter which one of the two main roads one chooses for implementing change. These are presented in the coming sections. 


\subsection{The role of leadership}

The role of leadership is independent of which main road one chooses to take. Leaders must lead through visions and overall goals. The larger the group/organization is, the more important it becomes. One of the reasons that this is so universal is that it provides space for the group and its members to use their individual creativity to solve the tasks. It uses the collective expertise of the group. However, it requires that the leader, e.g.:

- Is willing to join and show how tasks must be carried out (if required). Moreover, the leader has to be so knowledgeable that he or she, if needed, can act as a role model. Notably, this does not mean that the leader should do all the work for the employees.

- Serve as the creator of contacts, not as problem solver.

- Work with the monitoring of agreed activities and targets. Send feedback and let the person who made the job receive recognition for the accomplishment. Focus on those in the group who have the ability to influence.

- Work with action plans developed by those who must do the job. Do not let the action plans run over a too long period of time. Half a year may often be a good time horizon. Make sure to keep activity plans short and prioritize what is most important. Working with gross list and make decisions on new items on the list if space is available. Follow up about this on a regular basis, e.g twice a month: provide support, feedback and encouragement when the data is completed. Be sure that the agreements on completion dates are kept. Measure and visualize the number of items completed on time. If events are "slipping", find the root cause to this. In principal, this is a leadership issue. However, it may also be due to that the employee does not fit for the specific assignment or lacks time. In general, it is not due to unwillingness from the employee.

- Create conditions for a rapid feedback of the key indicators chosen to measure. Use them to guide and evaluate the work. The feedback often needs to be done weekly or whether it is possible, in real time.

- Try to create a positive atmosphere where "anything is possible". A positive spiral. It is therefore important to ensure that "easy victories", particularly early in the process, are achieved.

- When one set goals and prioritize activities always base this on the organization's value stream flow. It is the value stream which should be improved and not primarily single processes. It is only from the basis of the organization's value flow, ones effort can be measured correctly. Working with energy in general may not provide much value, but rather to be effective in reducing waste.

- Put effort into understanding the system links. There is always a larger improvement potential in a system than in a single component or process. Let the need control demand.

- Develops a strategy for how the plant should be operated in the long run. Do this in terms of the desired operational strategy, and the desired technology strategy, and how the desired system strategy should look like. When done, one can be flexible, and make changes when available opportunities occur in the business. Streamlining systems and components are not so costly when a major change is to be made but may be very costly if done as an operational acticity, e.g. lead to production disruptions. 


\section{Conclusion}

This paper addresses that increasing industrial energy efficiency is not only about technology, but equally important concerns the diffusion and adoption of energy management practices. In particular, the three factors, culture, will and acceptance and recognition interact between each other and create the conditions one has in an organization, department and group. It is these challenges that the leader has to work with to achieve the expected results. Moreover, results may be achieved in principally two different ways, where method governing is the way advocated for in this paper as it builds structural capital in the organization. If one choose to work with method governing, there are some fundamental principles that should be followed, e.g. focus on the organization's value stream, normal situation, standardization and continuous improvements. It is also of importance to define the system's normal state. From that position, improvements may be carried out in small steps. If a manager follows these basic recommendations outlined in this paper, and allows employees to understand and see the connections, improved results are achieved in the long run. Energy management practices using these principals may lead to employees showing improvements far above what the manager or organization thought was possible.

The applied methodology was shown to contribute with increased knowledge on how energy management practices successfully can be carried. Further research in the field using the applied methodology is suggested.

In conclusion, a fully successful in-house management program is dependent on both sound leadership and adoption of sounds methods. If either one is lacking, the full embodied (energy efficiency) potential in the organization is not released.

\section{References}

[1] IPCC, 2007. Contribution of Working Group III to the Fourth Assessment Report of the Intergovernmental Panel on Climate Change. Summary for Policymakers. Retrieved October 8, 2007, from: http://www.ipcc.ch/SPM0405 07.pdf

[2] Thollander P, Ottosson M. An energy efficient Swedish pulp and paper industry exploring barriers to and driving forces for cost-effective energy efficiency investments. Energy Efficiency 2008;1(1):21-34.

[3] Rohdin P, Thollander P, Solding P. Barriers to and drivers for energy efficiency in the Swedish foundry industry. Energy Policy 2007;35(1):672-7.

[4] Rohdin, P., Thollander, P., 2006. Barriers to and Driving Forces for Energy Efficiency in the Non-energy Intensive Manufacturing Industry in Sweden. Energy;31(12):1836-44.

[5] Christoffersen, L.B., Larsen, A., Togeby, M., 2006. Empirical analysis of energy management in Danish industry. Journal of Cleaner Production;14(5):516-26.

[6] Caffal, C., 1996. Energy management in industry. Centre for the Analysis and Dissemination of Demonstrated Energy Technologies (CADDET). Analysis Series 17. Sittard, The Netherlands.

[7] Thollander, P., Ottosson, M., 2010. Energy management practices in Swedish energyintensive industries. Journal of Cleaner Production 18(2):125-133.

[8] Boulding, K.E., 1956. General System Theory - The Skeleton of Science. Management Science 2 (3): 197-208. 